\title{
Diseño y plan de implementación para el montaje de una Oficina de Gestión de Proyectos (OGP) en MYV Consultores Asociados S.A.
}

\author{
Design and implementation plan for the assembly of a Project Management Office (PMO) in \\ MYV Consultores Asociados S.A.
}

Fecha de entrega: 3 de junio 2019

Fecha de aceptación: 30 de agosto 2019

\section{José Fernando Jiménez, David Alejandro León, Juan Sebastián Mahecha, Jhon Mauricio Manco y Manuel Guillermo Pita}

Escuela de Administración de Negocios, Universidad EAN, Calle 127 B Bis No 71 A 43, Barrio Niza - Bogotá D.C., Colombia, jjimenez1345@universidadean.edu.co,dleongar1614@universidadean.edu.co,jmahecha8437@universidadean.edu.co, jmanco_1@universidadean.edu.co,mpitariv8566@universidadean.edu.co

MYV es una organización de consultoría proyectizada que no ha logrado estandarizar los resultados de sus proyectos ejecutados. Este estudio ha identificado el estado actual, determinando aspectos positivos y por mejorar. Implementar una Oficina de Gestión de Proyectos OGP es una práctica común a nivel mundial recientemente, generando ventajas competitivas. Se estudia el ciclo de vida y gestión de proyectos y portafolio, diferentes modelos de buenas prácticas, tipos y clasificaciones de OGP, la cultura organizacional es factor crítico de éxito. El estudio es de tipo mixto, con herramientas cuantitativas y cualitativas, tipos de estudio descriptivo y correlacional, para el diagnóstico del estado actual y plantear la propuesta de OGP adecuada. Se recolectaron datos para determinar el estado de gestión del portafolio, cultura organizacional, efectividad, prácticas y madurez de la gestión de proyectos. Los resultados fueron valorados estadisticamente obteniendo efectos interesantes y grandes oportunidades de mejora, alta correlación entre las variables; igualmente, se determinó que la madurez en la gestión tiene grado de conocimiento básico, con avances en metodología propia y mejora continua, que deben ser complementados, con futuro prometedor en desarrollo, de continuar con el proceso hasta ahora seguido. Como resultado se plantea la implementación de OGP estratégica, cuyo grado de madurez se logre en un plazo máximo de dos años, planteando mapa estratégico de OGP y propuesta de modificación de la estructura organizacional y de la OGP, así como el plan de implementación en tiempos, métricas, riesgos y costos asociados. Se responde las preguntas planteadas y proyecta una OGP ajustada a la cultura organizacional encontrada.

Palabras clave: Oficina de Gestión de Proyectos OGP, gerencia de proyectos, mejores prácticas, objetivos estratégicos, cultura organizacional, gestión de portafolio, efectividad, madurez
MYV is a projected consulting organization, which has not been able to standardize the results of its executed projects. This document has identified the current status, determining positive aspects and improving the management of projects. To implement a project management office PMO is a recently worldwide practice; generating competitive advantages. The life cycle and the management of projects and portfolio were studied. The research carried out is of mixed type, with quantitative and qualitative tools, types of study descriptive and correlational, to develop the diagnosis of the current state to propose the appropriate PMO. Data was collected to determine the management status of portfolio, organizational culture, effectiveness, practices and maturity of project management. The results obtained were statistically assessed, obtaining interesting effects and great opportunities for improvement. Likewise, it was determined that the maturity in the project management has a basic knowledge degree, with advances in own methodology and continuous improvement, which must be complemented, with a promising future in development to continue with the process followed. Based on the foregoing, the implementation of a strategic PMO proposed, whose degree of maturity is reached within a maximum period of two years, with a strategic map of the PMO and a proposal for modification of the organizational structure and PMO, as well as the implementation plan in times, metrics, risks and associated costs. It is concluded that the research work carried out answers the questions posed and projects an PMO adjusted to the organizational culture found.

Keywords: Project Management Office PMO, project management, best practices, strategic objectives, organizational culture, portfolio management, effectiveness, maturity 


\section{Introducción}

La gestión de proyectos no es una práctica nueva, desde la época antigua esta actividad se ha convertido en una forma útil de cumplir los objetivos de proyectos y organizaciones a diferente nivel; poniendo en práctica tecnologías, conocimiento y herramientas conocidas o desarrollados para tal fin. Recientemente las organizaciones, que enfocan la ejecución de su objeto social por proyectos, se han preocupado cada vez más por implementar buenas prácticas que les permitan tener mejores resultados. Estas prácticas se han ido formalizando cada vez más, el diseño e implementación de una Oficina de Gestión de Proyectos OGP que centralice estas actividades a nivel de organización, ha generado una mejora sustancial en este desarrollo, mejorando sustancialmente los resultados obtenidos de la ejecución de los proyectos a su cargo, minimizando las probabilidades de fallos por causas imputables al proceso.

MYV es una empresa dedicada a la consultoría en diferentes temas asociados a la infraestructura física. Esta organización tiene dificultades para estandarizar los resultados de los proyectos ejecutados, el diseño e implementación de la OGP la impactará, obteniendo una ventaja competitiva respecto de otras organizaciones que no la tienen.

Esta investigación tiene como objetivo asimilar el conocimiento relevante actual en OGPs, de la misma forma se busca determinar el nivel de madurez en diferentes aspectos relacionados de MYV Consultores Asociados S.A., lo que determinará la propuesta más adecuada de OGP y su plan de implementación en la organización estudiada. Además, este trabajo busca diseñar y plantear el plan de implementación de la OGP adecuada al tamaño, actividad, cultura y necesidad de MYV.

\section{Oficinas de Gestión de Proyectos OGPS}

Dentro de las diferentes definiciones sobre las oficinas de gestión de proyectos OGPs, la definición más conveniente depende del grado de madurez de cada empresa y las posibles variaciones de tamaños, funciones, roles e importancia dentro de organización. Hill (2008) las define como un integrador de negocio que integra personas, procesos (metodologías y buenas prácticas) y herramientas para gestionar o influenciar el rendimiento de los proyectos; además, las considera como un facilitador para el entendimiento, aplicación de prácticas relacionadas con proyectos y la integración de los intereses de negocio de las empresas. Por otro lado, PMI (2017) y Hobbs y Aubry (2010), se centran en las características y atributos de estas, buscando una definición que incluya las relaciones que la oficina de proyectos debe tener con el entorno de la organización y la importancia de la comunicación, ya que estos son factores claves que impactan en el desempeño eficiente de la misma. A partir del entendimiento de que las OGPs son estructuras dinámicas y se desenvuelven en diferentes contextos organizacionales, existen diferentes tipos y niveles de oficinas de gestión de proyectos, las cuales reúnen diversas características que van desde el control básico y la gestión de prácticas para el desarrollo de proyectos hasta la alineación estratégica de los proyectos y los objetivos organizacionales. La Tabla 1 resume diferentes clasificaciones de OGPs.

Tabla 1: Clasificaciones de OGPs

\begin{tabular}{|c|c|c|c|c|}
\hline Hill (2008) & $\begin{array}{c}\text { Crawford } \\
\text { y Cabanis- } \\
\text { Brewin } \\
(2005)\end{array}$ & $\begin{array}{l}\text { PMI } \\
(2017)\end{array}$ & $\begin{array}{l}\text { Kerzner } \\
(2015)\end{array}$ & $\begin{array}{c}\text { Casey y Peck } \\
(2001)\end{array}$ \\
\hline $\begin{array}{l}\text { Centro de } \\
\text { excelencia }\end{array}$ & \multirow[b]{2}{*}{ Estratégica } & \multirow[b]{2}{*}{ Directiva } & \multirow[b]{2}{*}{ Estratégica } & \multirow[b]{2}{*}{$\begin{array}{l}\text { Pool de } \\
\text { recursos }\end{array}$} \\
\hline $\begin{array}{l}\text { Oficina de } \\
\text { gestión de } \\
\text { proyectos } \\
\text { avanzada }\end{array}$ & & & & \\
\hline $\begin{array}{l}\text { Oficina de } \\
\text { gestión de } \\
\text { proyectos } \\
\text { estándar }\end{array}$ & \multirow{2}{*}{$\begin{array}{l}\text { Unidad de } \\
\text { Negocios }\end{array}$} & \multirow{2}{*}{ Control } & \multirow{2}{*}{$\begin{array}{l}\text { Especiali- } \\
\text { zada }\end{array}$} & \multirow{2}{*}{$\begin{array}{l}\text { Torre de } \\
\text { Control }\end{array}$} \\
\hline $\begin{array}{l}\text { Oficina de } \\
\text { gestión de } \\
\text { proyectos } \\
\text { básica }\end{array}$ & & & & \\
\hline $\begin{array}{l}\text { Oficina de } \\
\text { proyectos }\end{array}$ & $\begin{array}{c}\text { Oficina } \\
\text { Control de } \\
\text { Proyectos }\end{array}$ & Aроуо & Tradicional & $\begin{array}{l}\text { Estación } \\
\text { meteoroló- } \\
\text { gica }\end{array}$ \\
\hline
\end{tabular}

\section{Cultura organizacional en el ámbito de proyectos}

Somos participes y espectadores de una sociedad, cuya evolución nos ha llevado a un frenético ritmo de vida y nos encontramos inmersos en un mercado laboral cada vez más competitivo, esto nos exige estar a la par de esta tendencia tomando la cultura organizacional como herramienta fundamental en este proceso, la cultura organizacional la podemos definir como la identidad de una organización, sus costumbres, su ADN. Ahora bien, en 
términos técnicos podemos encontrar definiciones como la de Schein (1992) en donde la cultura organizacional es el patrón de premisas básicas que un determinado grupo inventó, descubrió o desarrolló en el proceso de aprender a resolver sus problemas de adaptación externa $\mathrm{y}$ de integración interna y que funcionaron suficientemente bien a punto de ser consideradas válidas $\mathrm{y}$, por ende, de ser enseñadas a nuevos miembros del grupo como la manera correcta de percibir, pensar y sentir en relación a estos problemas. Para poder dimensionar la importancia de la cultura organizacional en el ámbito de proyectos, es necesario entender el entorno en el que se encuentra la organización y sus componentes, los cuales abarcan la integración organizacional, el relacionamiento de los diferentes interesados, el aprendizaje y gobernabilidad cultural en el ámbito de proyectos (Ariza, 2017a).

\section{Efectividad en la gestión de proyectos}

Uno de los conceptos a tener en cuenta en el ámbito de la gestión de proyectos es la efectividad. Este término va mucho más allá de la gestión adecuada para conseguir el desarrollo del proyecto con los recursos apropiados en el tiempo proyectado, implica también diversas técnicas de gestión que ayuden a los equipos a definir, gestionar y realizar el trabajo para alcanzar los mejores resultados posibles. La efectividad en la gestión de los proyectos implica la coordinación de una serie de actividades y asignación de recursos para adelantarlas, integrando el trabajo de un grupo de personas intervinientes en la concepción, estructura, desarrollo y ejecución del proyecto, aun cuando sus expectativas puedan ser diferentes, valorando su efectividad de modo distinto, de acuerdo con el rol o la relación que tienen con los proyectos. Es claro que para determinar la efectividad de la gestión realizada en los proyectos, se debe establecer un método de control que permita evaluar constantemente a medida que avanza la ejecución del proyecto, el proceso lógico del progreso de todas sus actividades, ubicándolos en tres categorías principales relacionados con la organización, la disciplina de gestión de proyectos y los resultados de los proyectos (Ariza, 2017a). De esta forma se pueden establecer factores que permitan identificar la articulación de las diversas actividades, y la dependencia de cada uno de los aspectos mencionados, para obtener una medición integral de la efectividad del proyecto. La efectividad de los proyectos ha sido medida en términos de los resultados de los proyectos, con dos orientaciones diferentes; la primera relacionada con el cumplimiento de los objetivos de los proyectos en cuanto a alcance, tiempo, costo y calidad. Y la segunda, refiriéndose a la satisfacción de los stakeholders internos y externos en términos del cumplimiento de los requerimientos, la utilidad de los resultados percibida por los clientes y la satisfacción del equipo con su participación en el proyecto (Ariza, 2017a).

\section{Madurez de gestión de proyectos a nivel organizacional}

Los modelos de madurez de gestión de proyectos son herramientas de gran valor con las que cuentan las organizaciones para identificar el estado actual en cuanto a la adopción de prácticas de gestión asociadas a los proyectos, procesos y su respectivo desempeño. Básicamente son marcos de referencia que describen el estado actual y el progreso ideal hacia la mejora o estado deseado pasando por etapas o niveles secuenciales (Man, 2007). Dentro de los diferentes modelos de madurez de gestión de proyectos, se pueden encontrar modelos como el OPM3 propuesto por PMI (2008), el PMMM de Kerzner (2002) o el modelo de madurez de Berkeley (Kwak e Ibbs, 2005). El Organizational Project Management Maturity Model OPM3 es un marco de referencia que provee una amplia visión organizacional de la gestión de portafolio, programas y proyectos para ayudar a la consecución de mejores prácticas en esos ámbitos. Este marco de referencia del OPM3 está hecho a partir de tres componentes interrelacionados: mejores prácticas, capacidades y resultados (PMI, 2008). El ciclo de implementación del OPM3 consiste en tres elementos entrelazados, conocimiento, evaluación y mejora.

En el caso del Project Management Maturity Model PMMM, es un modelo de medición de madurez el cual considera que la madurez se encuentra en el desarrollo de sistemas y procesos que por su naturaleza son repetitivos y a partir de esto garantizan una alta probabilidad de éxito lo que constituye las bases para alcanzar la excelencia en gestión de proyectos (Kerzner, 2002). El Modelo de Madurez en Gestión de Proyectos de Berkeley o Project Management Process Maturity Model es un modelo de madurez totalmente integrado, para medir, localizar, y comparar el actual estado de madurez en gestión de 
proyectos. El objetivo de este modelo es motivar a las organizaciones y a las personas a alcanzar niveles más altos y sofisticados de madurez en gestión de proyectos. El rango de los niveles de madurez del modelo va desde el uno (bajo) hasta el cinco (alto) usando una escala de Likert (Kwak e Ibbs, 2000). En el nivel uno o etapa adhoc no hay procesos, prácticas o datos de la gestión de proyectos en la organización, en el nivel dos o etapa de planificación se definen de manera informal lo problemas y procesos de la gestión de proyectos y se recopilan datos y analizan de manera informal, en el nivel tres o etapa gestionada, ya existe una gestión formal de los sistemas de planificación y control de los proyectos, los datos de la gestión de proyectos se gestionan de manera formal; en el nivel cuatro o etapa integrada la organización ya cuenta con un modelo de gestión de múltiples proyectos, se trabaja sobre la integración de procesos y datos y toda la data es analizada cuantitativamente, se miden y almacenan y por último, en el nivel 5 o etapa sostenida, todos los procesos de gestión de proyectos están en constante mejoramiento y se entienden completamente.

\section{Metodología de investigación y datos}

Se realiza una investigación de tipo mixto, que utiliza herramientas cualitativas y cuantitativas, las cuales contemplaron dos objetivos principales, desarrollar un diagnóstico de la situación actual en la organización MYV y tener los elementos necesarios para desarrollar la propuesta de la oficina de proyectos OGP. A su vez la investigación plantea dos tipos de estudio, en primer lugar, un estudio descriptivo consistente en llegar a conocer las situaciones, costumbres y actitudes predominantes en la organización a través de la descripción exacta de las actividades, objetos, procesos y personas. En segundo lugar, un estudio correlacional que determina si dos variables están correlacionadas o no (Hernández, 2017).

El objetivo del estudio de caso fue el de investigar la pertinencia, relevancia e impacto de la implementación de una oficina de proyectos en la compañía MYV, organización dedicada, principalmente, a la consultoría en aspectos de infraestructura tanto pública como privada. La Tabla 2 resume el grupo de colaboradores de MYV.

MYV es una empresa joven con amplia experiencia. MYV es una organización que funciona por proyectos que ejecuta en tres Unidades Estratégicas de Negocio UEN: infraestructura, jurídica y social, logrando implantarlas e integrarlas en la ejecución de sus proyectos de diseño, estructuración, interventoría y gerencia de obras de infraestructura para entidades de orden público y privado, permitiendo un mayor control en la ejecución, garantizando un resultado acorde a las necesidades de sus clientes.

Tabla 2: Grupo de colaboradores de MYV

\begin{tabular}{|l|c|c|}
\hline EJECUTIVO & 5 & $21.74 \%$ \\
\hline DIRECTORES & 3 & $13.04 \%$ \\
\hline PROFESIONALES & 11 & $47.83 \%$ \\
\hline TECNÓLOGOS & 3 & $13.04 \%$ \\
\hline APOYO GENERAL & 1 & $4.35 \%$ \\
\hline TOTALES & 23 & $100.00 \%$ \\
\hline
\end{tabular}

El periodo de tiempo en el cual se desarrolla el estudio es el comprendido entre los meses de junio de 2018 y culminando en el mes de abril de 2019. Para la recolección de los datos las técnicas utilizadas fueron variadas, para la gestión de portafolios se utilizó la entrevista tipo semiestructurada definida por Ariza (2017a). La entrevista se compone de un total de 25 preguntas divididas en dos secciones, la primera un total de 18 preguntas relacionadas con las prácticas de gestión del portafolio de proyectos, y la segunda un total de 7 preguntas relacionadas con la cultura organizacional asociada a la gestión del portafolio de proyectos, a través de las cuales se identificó la forma como la organización maneja el conjunto de proyectos que responden a su estrategia.

La segunda técnica de investigación utilizada fue la encuesta, la cual fue practicada a 16 de los 22 colaboradores de la organización, segmentados en tres grupos diferentes de stakeholders. Grupo A conformado por 6 personas que participan en un proyecto donde MYV realiza acompañamiento en aspectos técnicos y realiza la gerencia de proyecto en la interventoría. Un grupo B conformado por 4 personas que participan de un contrato de interventoría a la ejecución de instituciones educativas, para el Fondo de Financiamiento para las Instituciones Educativas FFIE con recursos del Ministerio de Educación Nacional. Un último grupo de 6 personas hacen parte del staff de MYV y participan en algunos o todos los proyectos que se desarrollan en la organización. 
Estos tres grupos de stakeholders representan la realidad de ejecución de proyectos en la organización: i) personas que laboran en proyectos no gestionados directamente, ii) personas que laboran en proyectos gestionados directamente, y iii) personas que laboran en el staff que apoya a todos o parte de los proyectos en ejecución, desde la concepción de la idea o decisión de participación en el proceso, etapa precontractual, etapa contractual o de ejecución, etapa de liquidación o post contractual y etapa de vigencia de las garantías.

Se utiliza una segunda encuesta para realizar la medición de la efectividad de la gestión de proyectos, utilizando la misma muestra del punto anterior (16 personas asociadas a la ejecución de los proyectos en MYV). De igual manera, se utilizó un constructo de efectividad de la gestión de los proyectos, que fue validado previamente desde el punto de vista estadístico. Se utilizó la misma encuesta presentada por Ariza (2017b), como medio de recolección de datos, la cual se compone de 11 indicadores que buscan medir la percepción de los colaboradores respecto a este tema. Otra técnica utilizada en esta investigación fue la auditoría de proyectos con el objetivo de determinar la correspondencia de la aplicación de los criterios de las prácticas de gestión de proyectos acordes a la guía PMBOK del PMI (2017), específicamente los cinco grupos de proceso (inicio, planeación, ejecución, monitoreo y seguimiento y cierre), se realizó una auditoria a 3 proyectos ejecutados por MYV, el primero no gestionado directamente en consorcio, otro gestionado directamente en consorcio y el tercero ejecutado directamente.

La última técnica para recolección de datos utilizada es el modelo de Kezner para medir la madurez de gestión de proyectos en MYV. Este modelo considera todos los aspectos de la compañía, desde las relaciones de trabajo entre empleados y los directivos, las distintas funciones de todos los participantes en los proyectos en especial el papel de los patrocinadores de proyectos, y los aspectos de cultura y activos de la organización (Kerzner, 2002), estableciendo cinco niveles de madurez para la gestión de proyectos que se identificarán progresivamente en la organización a partir de su aplicación en la organización.

\section{Resultados del caso de estudio Gestión del portafolio de proyectos}

Teniendo en cuenta que la organización investigada no cuenta con manejo formal de portafolio, se realizó la entrevista a tres personas que cumplen labores como encargados del portafolio. Estas entrevistas fueron realizadas en octubre de 2018 y validadas, como confirmación, en enero de 2019. De la información obtenida se sintetizan los resultados que se presentan a continuación.

1. Aunque no se cuenta con una definición formal de portafolio de proyectos, a lo largo de las diferentes iniciativas se aplican prácticas asociadas tanto a la gestión de proyectos como a la gestión de portafolios.

2. Se encontró que el gerente de la unidad de infraestructura asume el rol de administrador del portafolio.

3. No se tienen mecanismos, políticas o espacios formales de comunicación del estado de los proyectos en curso a la empresa, nuevos proyectos o proyectos finalizados.

4. Se desarrolla la identificación inicial de riesgos legales, normativos, técnicos, etc., registrándolos, en pocas ocasiones se actualizan y/o monitorean a lo largo del proyecto.

5. No se maneja formalmente el concepto de patrocinador ni a nivel de portafolio ni a nivel de proyectos.

6. Aunque hay ausencia de prácticas formales de gestión de portafolio en la empresa, los proyectos están alineados con los objetivos estratégicos de la compañía y por lo general los esfuerzos realizados en las iniciativas aportan valor al cumplimiento de metas organizacionales.

7. La empresa tiene debilidades en el proceso de evaluación de proyectos y levantamiento de requerimientos, lo que eventualmente genera dificultades en la identificación y seguimiento de los beneficios tangibles e intangibles de los proyectos a la estrategia.

8. No se cuenta con procedimientos formales ni herramientas para la estimación y asignación de recursos a los proyectos, así como al seguimiento de su disponibilidad, su asignación se realiza de acuerdo a la percepción y criterio del gerente de proyectos. 
9. La organización se centra en el cumplimiento y medición de las métricas contractuales de servicio y calidad con cada cliente; pero tiene debilidad en la medición de indicadores asociados al desempeño de los proyectos a nivel de costos, tiempo y riesgos.

10. Se desperdicia conocimiento colectivo al no registrar de forma continua las lecciones aprendidas y buenas prácticas identificadas en cada iniciativa.

\section{Cultura organizacional y efectividad de la gestión de los proyectos}

Se hizo la valoración del grado de confiabilidad general del instrumento aplicando el coeficiente alfa de Cronbach (Hernández et al., 2014). La valoración se realizó en cuatro instancias diferentes, encontrando en todas las aplicaciones que el resultado indica que la confiabilidad es muy alta, valores mayores a 0.7 se consideran aceptables en este tipo de investigaciones (Hair et al., 2013). Igualmente se determinó que los datos obtenidos en la muestra se distribuyen normalmente.

La cultura organizacional está compuesta por ocho dimensiones: 1) Alineación Estratégica de Proyectos AEP, 2) Entendimiento en el Ámbito de Proyectos EAP, 3) Adopción de Prácticas de Gestión de Proyectos APGP, 4) Valoración del Conocimiento Asociado a los Proyectos VCAP, 5) Cohesión Entre Stakeholders CES, 6) Empoderamiento E, 7) Consideración con los Stakeholders CCS, 8) Adaptación para la Solución de Problemas ASP y puntaje final Cultura Organizacional CO. La Figura 1 presenta los promedios obtenidos para cada variable.

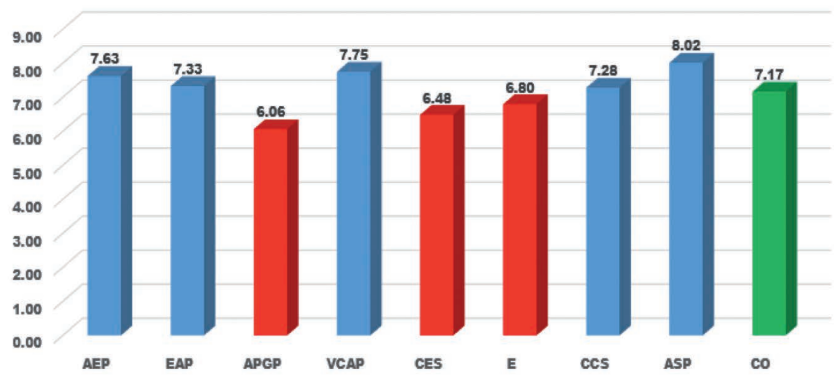

Figura 1: Valoración cultura organizacional en MYV

Realizando el análisis se puede determinar que se debe realizar un trabajo fuerte para mejorar las valoraciones recibidas en todas las dimensiones evaluadas, el promedio general de Cultura Organizacional es de 7.17. En las dimensiones que más se debe trabajar, debido a que son las que tienen más baja valoración, APGP, CES, E, por debajo del promedio general de $\mathrm{CO}$. Aunque las otras dimensiones tienen valoraciones promedio mayores, también deben ser trabajadas, implementando una mejora continua en ellos.

La efectividad de los proyectos está compuesta por tres dimensiones: 1) Cumplimiento de Expectativas CE, 2) Satisfacción del Equipo de Proyecto SEP, 3) Logro de los Compromisos LC y puntaje final Efectividad de Proyectos EP. En la Figura 2 se muestran los promedios obtenidos para cada variable.

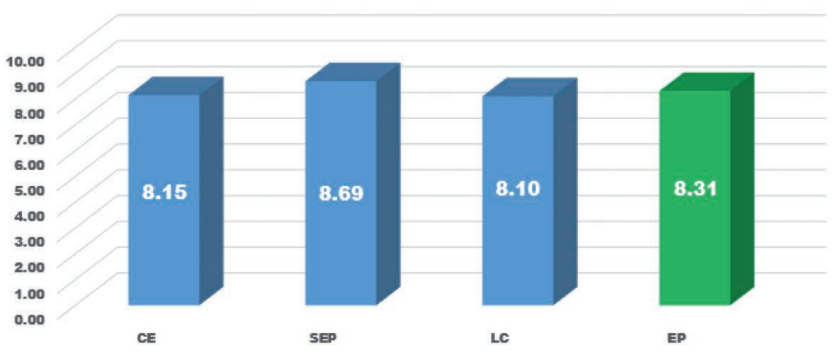

Figura 2: Valoración de la efectividad de proyectos en MYV

Se puede determinar que tanto las tres dimensiones como el promedio general obtenido para la efectividad de proyectos es superior a 8 , superior al $80 \%$, lo cual permite la implementación de mejoras en todos los aspectos. Es posible que se haya podido presentar un sesgo inherente al deseo de obtener aprobación social por parte de los encuestados que realizaron la valoración (Podsakoff et al., 2003). Para evitar este posible sesgo, se indicó a las personas que la información que entregaban era confidencial y que solo se harían públicos los resultados estadísticos.

\section{Análisis de correlación cultura organiza-cional}

La Figura 3 presenta el desarrollo de una matriz $(8 \times 8$ - 28 emparejamientos posibles) de correlación para la valoración de cultura organizacional en el ámbito de los proyectos. Los valores obtenidos fueron relacionados y analizados de acuerdo a los rangos determinados por Lewis-Beck et al. (2003), los cuales son de alta correlación superior o igual a 0.60 (verde), moderada correlación entre 0.30 e inferior a 0.60 (amarillo) y baja correlación valores menores a 0.30 (rojo - no hay). En la Tabla 3 se presentan 
las duplas (emparejamientos) con mayor correlación, de ellas se desarrolla un análisis explicativo sobre las más altas (las cuatro que tienen correlación superior a 0.90), que son las que mayor impacto tienen en la organización.

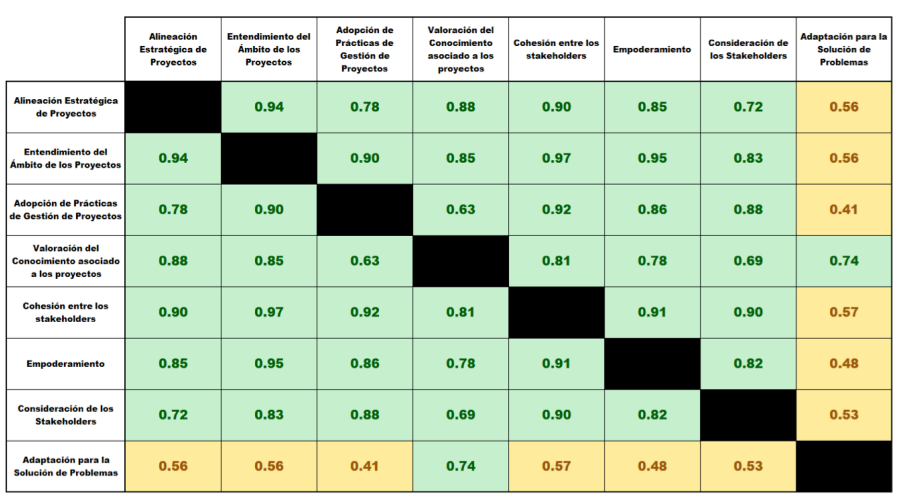

Figura 3: Matriz de correlación para la valoración de cultura organizacional

Tabla 3: Correlaciones para la valoración de cultura organizacional

\begin{tabular}{|c|c|c|}
\hline \multicolumn{2}{|c|}{ VARIABLES CORRELACION ALTA } & PUNTAJE \\
\hline $\begin{array}{c}\text { Cohesión entre los } \\
\text { Stakeholders }\end{array}$ & $\begin{array}{c}\text { Entendimiento del } \\
\text { Ámbito de los Proyectos }\end{array}$ & 0.97 \\
\hline $\begin{array}{c}\text { Alineación Estratégica de } \\
\text { Proyectos }\end{array}$ & $\begin{array}{c}\text { Entendimiento del } \\
\text { Ámbito de los Proyectos }\end{array}$ & 0.94 \\
\hline $\begin{array}{c}\text { Cohesión entre los } \\
\text { stakeholders }\end{array}$ & $\begin{array}{c}\text { Adopción de Práctica de } \\
\text { Gestión de Proyectos }\end{array}$ & 0.92 \\
\hline $\begin{array}{c}\text { Empoderamiento } \\
\text { Cohesión entre los } \\
\text { Stakeholders }\end{array}$ & 0.91 \\
\hline $\begin{array}{c}\text { Cosideración de los } \\
\text { Stakeholders }\end{array}$ & $\begin{array}{c}\text { Empoderamiento } \\
\text { Cohesión entre los } \\
\text { Stakeholders }\end{array}$ & $\begin{array}{c}\text { Conocimiento asociado a } \\
\text { los Proyectos }\end{array}$ \\
\hline $\begin{array}{c}\text { Valoración de } \\
\text { Adaptación para la } \\
\text { Solución de Problemas }\end{array}$ & $\begin{array}{c}\text { Conocimiento asociado a } \\
\text { los Proyectos }\end{array}$ & 0.74 \\
\hline
\end{tabular}

Existe cohesión entre los stakeholders y un entendimiento del ámbito de los proyectos porque se evidencia una cultura propia de la organización enfocada al espíritu colaborativo, de ahí que las áreas que intervienen y apoyan el proyecto funcionan como un equipo y no como células de trabajo. Existe alineación estratégica de proyectos y un entendimiento del ámbito de los proyectos porque los proyectos son referenciados como parte de la estrategia organizacional, se busca sinergia entre ellos en busca de optimización de recursos. Existe cohesión entre los stakeholders y adopción de prácticas de gestión de proyectos porque el personal en MYV a través del tiempo adoptó prácticas de manera empírica que le permiten ser eficientes en su operación. La implementación de una metodología permitirá ser más eficiente en desarrollo, implementación, seguimiento, monitoreo y mejora continua de sus procesos. Existe empoderamiento y cohesión entre los stakeholders porque el personal MYV tiene una estructura jerárquica leve, pero que no tiene limitaciones de empoderamiento del equipo, ya que todas las personas tienen el conocimiento y experticia para desarrollar sus labores de manera dinámica y proactiva.

\section{Análisis de correlación efectividad de los proyectos}

La Figura 4 presenta el desarrollo de una matriz ( 3 x 3 - 3 emparejamientos posibles) de correlación para la valoración de efectividad de los proyectos.

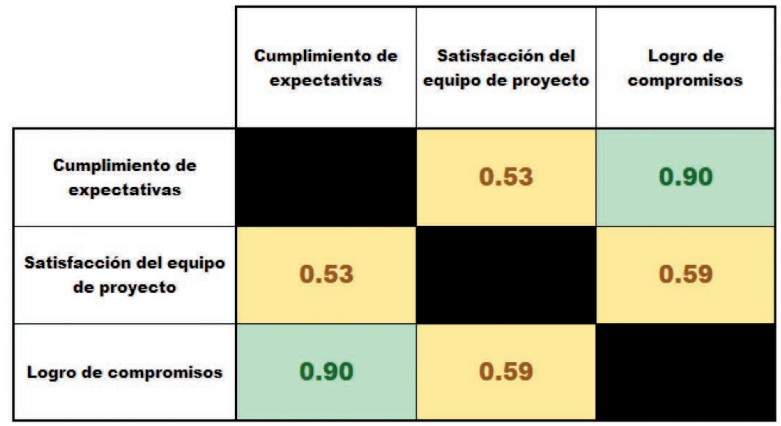

Figura 4: Matriz de correlación de efectividad de proyectos

Los valores obtenidos fueron relacionados y analizados de acuerdo a los rangos determinados por Lewis-Beck et al. (2003), los cuales son de alta correlación superior o igual a 0.60 (verde), moderada correlación entre 0.30 e inferior a 0.60 (amarillo); y baja correlación valores menores a 0.30 (rojo - no hay). La relación y nivel de dependencia entre las dimensiones de efectividad de los proyectos en MYV es alta y la variación de una dimensión impactará en las demás con impactos medios o altos. En la Tabla 4 se presentan las duplas (emparejamientos) con mayor correlación, de ellas se desarrolla un análisis explicativo para la más alta.

Existe cumplimiento de expectativas y logro de compromisos porque los equipos de los proyectos enfocan sus esfuerzos hacia la consecución de las metas, y a su vez, se preocupan por satisfacer los resultados esperados de los diversos stakeholders del proyecto y de la organización. 
Tabla 4: Correlaciones para la efectividad de proyectos

\begin{tabular}{|c|c|c|}
\hline \multicolumn{2}{|c|}{ VARIABLES } & PUNTAJE \\
\hline $\begin{array}{c}\text { Cumplimiento de } \\
\text { expectativas }\end{array}$ & Logro de compromisos & 0.90 \\
\hline $\begin{array}{c}\text { Satisfacción del equipo } \\
\text { de proyecto }\end{array}$ & Logro de compromisos & 0.59 \\
\hline $\begin{array}{c}\text { Cumplimiento de } \\
\text { expectativas }\end{array}$ & $\begin{array}{c}\text { Satisfacción del equipo } \\
\text { de proyecto }\end{array}$ & 0.53 \\
\hline
\end{tabular}

La organización refuerza el hecho de que las personas expresen sus opiniones abiertamente para llegar a acuerdos en los proyectos. En los proyectos se busca solucionar los problemas rápidamente para poder avanzar. Cuando hay conflictos en los proyectos, se trata de asumir la posición colectiva que más convenga para los intereses del proyecto. Las áreas de la organización negocian entre sí para lograr los objetivos de los proyectos. La organización promueve el análisis y entendimiento de la posición de los stakeholders frente a los proyectos y de su grado de interés o desinterés en ellos. En los proyectos siempre se consulta a las personas que pueden ser afectadas por el proyecto y su opinión es tenida en cuenta en las decisiones que se toman. En los proyectos se busca comprender las necesidades de los stakeholders y se definen los requerimientos acordes a estas necesidades. En los equipos de proyecto se considera a los stakeholders externos (proveedores, contratistas, clientes) como aliados con quienes se comunican activamente.

\section{Relación cultura organizacional en el ámbito de los} proyectos y efectividad de los proyectos

La Tabla 5 muestra la correlación para la valoración de cultura organizacional en el ámbito de los proyectos y efectividad de los proyectos.

Tabla 5: Correlación de la cultura organizacional en el ámbito de los proyectos y efectividad de los proyectos para la efectividad de proyectos

\begin{tabular}{|c|c|c|}
\hline \multicolumn{2}{|c|}{ VARIABLES } & PUNTAJE \\
\hline Cultura organizacional & $\begin{array}{c}\text { Efectividad de los } \\
\text { proyectos }\end{array}$ & 0.90 \\
\hline
\end{tabular}

Esta alta correlación encontrada refiere que para MYV la efectividad de los proyectos es parte de su cultura organizacional, lo que claramente evidencia que la organización sobre la cual se desarrolla la presente investigación tiene fuertes rasgos e interés en el cumplimiento de sus compromisos contractuales, que ha elevado a cultura empresarial.

\section{Regresión lineal}

Con los resultados obtenidos en las encuestas aplicadas y la correlación expuesta del 0.90 entre cultura organizacional en el ámbito de los proyectos y efectividad de los proyectos, se procedió a realizar la regresión lineal entre las dos variables, de esta manera se generó un modelo matemático que permite proyectar y entender la forma en la que se podría impactar la efectividad de los proyectos cuando se presente, planee o ejecute una modificación en la cultura organizacional en el ámbito de los proyectos en MYV. Posterior a esto se realiza el análisis de regresión, obteniendo un coeficiente de determinación $\mathrm{R}^{2}=0.81$. Este valor indica el nivel en que se explica un cambio en la variable dependiente- $x$ (efectividad) por un cambio en las variables independientes, en este caso, las categorías culturales. Un coeficiente alto implica que la variación en la variable de efectividad se explica en un $80 \%$ por las categorías culturales. El valor crítico de $\mathrm{F}$ o $p=0$, es una medida de significación estadística, al ser cero se rechaza la hipótesis nula de correlación. La mayoría de los residuos tienden a cero, razón por la cual se infiere que están completamente alienados con el modelo.

\section{Prácticas de gestión de los proyectos}

Con el objetivo de determinar la correspondencia de la aplicación de los criterios de las prácticas de gestión de proyectos acordes a la guía PMBOK del PMI (2017), se realizó una auditoria a 3 proyectos ejecutados por MYV, uno no gestionado directamente en consorcio, otro gestionado directamente en consorcio y el tercero ejecutado directamente; para ello se utilizó la lista de chequeo de gestión del alcance, tiempo, costo, calidad, riesgos, adquisiciones y contrataciones, stakeholders, recursos humanos, comunicaciones y cambio en los proyectos (Ariza, 2017b).

En relación a la gestión del alcance, se aplican los procesos de gestión necesarios, aunque se encuentran oportunidades de mejora respecto a involucrar a los stakeholders al momento de definir los requerimientos del proyecto. Las prácticas de gestión del cronograma se aplican formalmente en los proyectos auditados. En los dos proyectos realizados mediante consorcio, se identificaron posibilidades de 
mejora relacionadas con el monitoreo de las desviaciones del cronograma y la determinación de las acciones a tomar cuando se presentan. Se determinó gran relevancia en la gestión del presupuesto, identificándose como factor de alta importancia en la gestión de los proyectos ejecutados por la organización. En los proyectos realizados mediante consorcio, se identificaron posibilidades de mejora en la verificación de las métricas de calidad y su correspondencia con las metas esperadas; así como en la obtención de la aceptación formal de los resultados y entregables de los proyectos. Se encontró que la gestión de los riesgos se aplica de manera aceptable. En los proyectos realizados mediante consorcio, se identificaron falencias en la definición de las respuestas a los riesgos según su criticidad, en el control de los riesgos identificados y la identificación de nuevos riesgos durante el desarrollo de los proyectos. En todos los proyectos auditados se encontró que se aplican prácticas adecuadas tanto en la definición de criterios, modos de contratación y selección de proveedores calificados. De igual manera, se tienen procedimientos y acciones de seguimiento al cumplimiento de los compromisos de los accionistas a través de la figura de interventoría.

Dentro de los ítems auditados, la gestión de stakeholders fue uno de los criterios con menor valoración sobre todo en los proyectos que son ejecutados en consorcio donde la ejecución es realizada por terceros. Existen debilidades de cara a la identificación de los involucrados en las iniciativas, así como en la medición y análisis de las percepciones de estos durante el desarrollo de los proyectos. En los proyectos auditados se evidenció la aplicación idónea de prácticas de gestión de los recursos humanos; ya que debido al tipo de iniciativas que maneja MYV, es indispensable contar con una definición clara de roles y responsabilidades. De igual manera, se realiza seguimiento al desempeño de los equipos y buscan condiciones adecuadas para tener un ambiente que fomente el desarrollo del trabajo de los equipos. En los proyectos auditados, especialmente en los ejecutados en consorcio y particularmente aquellos no gestionados directamente; se evidenciaron falencias en el proceso de gestión de las comunicaciones de los interesados ya que desde el inicio del proyecto en ocasiones no se definen requerimientos ni canales formales de comunicación ni se cuenta con estrategias robustas de comunicación de los avances del proyecto. Adicional a lo anterior, el registro y socialización de lecciones aprendidas presenta falencias ya que en muchas ocasiones no se realiza de manera adecuada y continua esta actividad. Para las iniciativas que son ejecutadas directamente por la organización se evidencia que se aplican de forma adecuada prácticas y estrategias para la gestión del cambio en sus proyectos, pero para el caso de los proyectos manejados en consorcio se evidenciaron falencias en este aspecto ya que no siempre se verifican y se hace seguimiento de los resultados de los cambios aprobados en los proyectos.

En resumen, se evidencian grandes posibilidades de mejora en todas las áreas de gestión de la organización. En proyectos ejecutados mediante figuras asociativas, consorcios principalmente y en especial cuando no son gestionados por la organización investigada, se encuentra mayor debilidad en algunos aspectos, razón por la cual se recomienda que, salvo que el(los) asociado(s) tenga(n) madurez empresarial sólida comprobada, se implementen procesos y procedimientos propios, lo que podría conllevar a tener mejoras sustanciales en algunas áreas.

\section{Medición de la madurez en la gestión de proyectos}

Para determinar la madurez en gestión de proyectos se utilizó como herramienta de análisis el instrumento propuesto Kerzner (2002), el cual sirve como soporte técnico para establecer un juicio de expertos a partir de los resultados obtenidos en cada grado o nivel de madurez en la gestión de proyectos que presenta la organización. El juicio de expertos fue realizado por cuatro de los cinco autores del presente documento, el quinto no participó en esta evaluación, toda vez que es el gerente de la unidad de infraestructura de la organización y podría generarse alguna visión sesgada de la situación. Para eliminar este posible sesgo, se retira, para este tema específico, la persona involucrada. Aplicados los instrumentos anteriores se obtuvo como resultado la información que se presenta a continuación para cada uno de los niveles de madurez.

\section{Nivel 1 lenguaje común (conocimiento básico)}

Para este nivel el instrumento divide en 8 categorías el total de las preguntas, dando a cada una la posibilidad de obtener 100 puntos. De su aplicación se obtiene una puntuación por categoría, la cual se muestra en la Tabla 6 . 
Tabla 6: Puntuación por categoría

\begin{tabular}{|c|c|}
\hline Gestión del alcance/ integración: & 100 \\
\hline Gestión del tiempo: & 80 \\
\hline Gestión del costo: & 70 \\
\hline Gestión de recursos humanos: & 40 \\
\hline Gestión de compras: & 90 \\
\hline Gestión de la calidad: & 30 \\
\hline Gestión de riesgos: & 90 \\
\hline Gestión de las comunicaciones & 90 \\
\hline Total & 590 \\
\hline
\end{tabular}

Según el instrumento de medición en aquella categoría que obtenga menos de 60 puntos, en ella existe una deficiencia y se debe trabajar en fortalecer los principios básicos necesarios en gestión de proyectos. Por otra parte, cuando el total de la puntuación no supera los 600 puntos el instrumento establece que podría existir núcleos de gestión de proyectos, cada núcleo puede tener un nivel de conocimiento diferente de la gestión de proyectos, y esta relación depende de qué núcleos son impulsados por los proyectos y cuáles no. Para el caso de la organización estudiada, por tratarse de proyectos ejecutados en su mayoría por alianzas estratégicas o por intermediación de terceros, es razonable que no posean el $100 \%$ de control sobre la gestión de recursos humanos y control de la gestión de la calidad en los proyectos, teniendo de presente que el alcance de autonomía y decisión sobre estos procesos está limitado al rol que desempeñe en la ejecución del proyecto.

\section{Nivel 2 procesos comunes}

Este nivel 2 es la etapa en la que una organización hace un esfuerzo concertado para utilizar la gestión de proyectos y para desarrollar procesos y metodologías para apoyar su uso efectivo. En este nivel la organización se da cuenta de que las metodologías comunes y los procesos son necesarios para que el éxito de la gestión de un proyecto pueda repetirse en otros proyectos.

Para el nivel 2 el instrumento de análisis establece 20 preguntas segmentadas en 5 categorías, que denomina fases de ciclo de vida, estas muestran el avance o retroceso en cada una de ellas llevándolas con una puntuación de -12 hasta +12 . A partir de 6 puntos en adelante se consideran puntos altos para una fase del ciclo de vida, e indican que estos valores evolutivos de fases de madurez precoz han sido alcanzados o por lo menos la organización se encuentra en evolución en esta fase. Cuando los números son muy bajos se establece que aún no se han alcanzado las fases. La Tabla 7 resume los resultados.

Tabla 7: Puntuación por categoría

\begin{tabular}{|l|c|}
\hline Fases del ciclo de vida & Puntos obtenidos \\
\hline Madurez & 2 \\
\hline Crecimiento & 9 \\
\hline Gerencia de línea & 0 \\
\hline Ejecutivos & 3 \\
\hline Embrionario & 7 \\
\hline
\end{tabular}

Este resultado indica que la organización está evolucionando considerablemente en las fases de crecimiento y embrionario, y las han alcanzado, sin embargo, para las fases de madurez y ejecutivos, no se alcanzan y su proceso evolutivo es lento, por último, la fase de gerencia de línea no ha comenzado su etapa evolutiva todavía. En conclusión, para el caso de estudio los procesos comunes aún están en etapa de graduación y es evidente el hecho de que ciertas expectativas de comportamiento progresivo del personal de la organización se requieren para la ejecución repetitiva de la metodología de gestión de proyectos efectiva.

\section{Nivel 3 metodología singular o metodología propia}

Este es el nivel en el que la organización reconoce que la sinergia y los procesos de control pueden lograrse mejor a través del desarrollo de una metodología singular en lugar de utilizar múltiples metodologías. En este nivel, la organización está totalmente comprometida con el concepto de gestión de proyectos, para ello el instrumento de análisis está compuesto de 42 preguntas de opción múltiple, segmentados en 6 áreas o componentes de la excelencia, que le permitirán a la organización confrontarse frente a otras organizaciones que han alcanzado este nivel de madurez, por medio de la puntuación obtenida, la cual es contrastada con la interpretación de los resultados que proporciona el instrumento (Tabla 8). 
Tabla 8: Puntuación por categoría

\begin{tabular}{|c|c|}
\hline Procesos Integrados & 24 \\
\hline Cultura & 27 \\
\hline Apoyo a la gestión & 27 \\
\hline Formación y educación & 13 \\
\hline Gestión informal de proyectos & 26 \\
\hline Excelencia conductual & 27 \\
\hline Total & 139 \\
\hline
\end{tabular}

El resultado corresponde con el rango entre 80-146, lo que significa que es probable que la compañía sólo esté hablando sobre la gestión de proyectos. El apoyo es mínimo. La compañía cree que es lo correcto para hacer, pero no ha descubierto los verdaderos beneficios o lo que ellos, los ejecutivos, deberían estar haciendo. La empresa sigue siendo una organización funcional.

Según estas mediciones la organización investigada se encuentra en el rango en el cual la organización hace muy poco por ejecutar los proyectos con un enfoqué estructurado en gestión de proyectos, su enfoque es mucho más funcional que proyectizada.

\section{Nivel 4 benchmarking}

En este nivel el benchmarking de la gestión de proyectos determina un proceso que busca comparar continuamente las prácticas de gestión de proyectos de la organización con las prácticas de los líderes en cualquier parte del mundo; su objetivo es obtener información que le ayude a mejorar su propia actuación. El instrumento establece 25 preguntas midiendo dos perspectivas, la primera si ¿la organización está realizando benchmarking? y, en caso afirmativo, ¿se está haciendo hincapié en la evaluación comparativa cuantitativa o cualitativa?. La Tabla 9 muestra los resultados de la aplicación del instrumento.

Tabla 8: Puntuación por benchmarking

\begin{tabular}{|l|c|}
\hline Benchmarking cuantitativo & 12 \\
\hline Benchmarking cualitativo & 8 \\
\hline Resultados combinados & 20 \\
\hline
\end{tabular}

Se determina en el benchmarking cuantitativo que se está iniciando su etapa de evaluación comparativa y no cuenta con un equipo para este proceso. Para el caso de benchmarking cualitativo la organización está a un nivel aceptable del proceso de desarrollo de cultura metodológica. Aunque no existe un equilibrio total entre los dos niveles de benchmarking, la diferencia no es relevante, lo que supone un buen indicador en la línea de evaluación comparativa.

\section{Nivel 5 mejora continua}

Este nivel busca que la organización evalúe la información aprendida durante el benchmarking e implementa los cambios necesarios para mejorar la gestión del proyecto. Es en este nivel que la empresa se da cuenta de que la excelencia en la gestión de proyectos es un viaje interminable. El instrumento de análisis establece 16 preguntas basadas en los cambios de mejora continua sólo en los últimos 12 meses, estableciendo rangos de medición de la puntuación obtenida. Para el caso estudio se obtuvo 22 puntos, lo que los ubica como una organización con iniciativa de búsqueda de formas de mejora continua, aunque no de manera proactiva y con algunos focos de resistencia al cambio.

\section{Evaluación general}

Con los resultados de la valoración del instrumento de medición de madurez de la gerencia de proyectos de cada nivel de Kerzner (2002), se determina que la organización se encuentra en un nivel de madurez de conocimiento básico, si bien es cierto que presenta avances en los niveles de metodología propia y mejora continua, estos no son muy significativos y en contraste con los bajos avances de la organización en los niveles de lenguaje común, procesos comunes y benchmarking, se presenta una etapa temprana de desarrollo de estrategias para implementar la gestión de proyectos en la organización. Las prácticas actuales de la organización en gestión de proyectos, los procesos y su desempeño determinan que su nivel de evolución está en pleno auge y puede darse progresivamente y de manera efectiva si se integran todos los integrantes de la organización y se siguen los parámetros que establece la gestión de proyectos. Como resultado final de la investigación se propone el diseño y plan de implementación de la Oficina de Gestión de Proyectos OGP, así como la transformación que debe tener la estructura de la organización, así como el periodo de tiempo de implementación y madurez. 


\section{Conclusiones}

De los hallazgos principales encontrados en esta investigación se resalta el resultado de la aplicación de la herramienta de madurez propuesta por Kerzner (2002), concluyendo que la empresa MYV a pesar de ser una empresa proyectizada, se encuentra en el nivel inferior del modelo de madurez denominado como conocimiento básico en donde se apropian y reconoce la importancia de tener una buena comprensión sobre los conocimientos, lenguaje y terminología asociada al ámbito de gestión de proyectos. Si bien dentro del análisis del nivel de madurez realizado a MYV se identifican avances en aspectos como metodología propia y mejora continua; se evidencian importantes brechas y oportunidades de mejora en los niveles de lenguaje común, procesos comunes y benchmarking.

Respecto a los factores que favorecen o dificultan la gestión de proyectos en la empresa, se identificó que a pesar que MYV centra su modelo de trabajo en la gestión de proyectos, la alta gerencia de la compañía concuerda en que no se tiene una definición formal del portafolio de proyectos, así como de un responsable formal y claramente definido que asuma el rol de administrador de los proyectos en curso y en etapa de formulación, este rol en muchas ocasiones es asumido por el gerente de la unidad de infraestructura. Dentro de las dimensiones de cultura organizacional, se encontró que MYV presenta los mayores puntajes en adaptación para la solución de problemas (8.02), valoración del conocimiento asociado a proyectos (7.75) y alineación estratégica de proyectos (7.63). Estas tres dimensiones deben ser consideradas por la organización como los principales habilitadores para promover las condiciones necesarias para incrementar el nivel de madurez de la organización de cara a la gestión de proyectos. Por otro lado, tres aspectos claves que debe fortalecer la organización son la adopción de prácticas de gestión de proyectos (6.06), cohesión entre los stakeholders (6.48) y el empoderamiento de los equipos de la organización asignados a las diferentes iniciativas (6.80). En cuanto a la medición de la efectividad de los proyectos en la organización y el respectivo análisis de las tres dimensiones propuestas por Ariza (2017b), se obtuvo una valoración superior a 8 en todas estas, concluyendo que, a pesar de no tener un modelo de madurez alto en gestión de proyectos, la filosofía y pilares de MYV están orientados por un lado al cumplimiento de expectativas y compromisos de las diferentes iniciativas, y por otro, a la satisfacción del equipo de proyecto. Sin embargo, se pudo haber presentado un sesgo inherente al deseo de obtener aprobación social por parte de los encuestados que realizaron la valoración. Se trató de evitar este sesgo, indicándole a las personas que la información que entregaban era confidencial y que solo se harían públicos los resultados estadísticos. Adicional a esto se identificó que, a pesar de tener ausencia de prácticas formales de gestión de portafolio en la empresa, los proyectos están alineados con los objetivos estratégicos de la compañía y por lo general los resultados de los diferentes proyectos aportan valor al cumplimiento de metas y objetivos de la empresa. Lo mencionado anteriormente, da respuesta al segundo objetivo específico de la investigación el cual tenía por fin la identificación de problemas, obstáculos y factores de éxito para la implementación de la OGP en la empresa.

Como parte de la respuesta al tercer objetivo específico, que buscaba identificar factores externos relacionados con la gestión de proyectos presentes en la industria en donde se desempeña la empresa, se identificó que uno de los factores que dificultan la gestión de proyectos en el sector de la construcción y consultoría, es que de manera reiterada, el rol de encargado de proyectos de manera parcial o informal recae principalmente sobre los equipos a cargo de la ejecución de las obras dando prioridad a la ejecución e implementación de la iniciativa; sin hacer énfasis profundo en la aplicación exhaustiva de buenas prácticas de gestión de proyectos. Respecto al nivel de madurez de MYV y sus necesidades y expectativas a corto, mediano y largo plazo, se propone la implementación de una OGP de carácter estratégico, la cual trabajará en torno a la conexión y fortalecimiento del vínculo entre la estrategia de la organización y la correcta gestión del portafolio de la misma. Dentro de las características de la OGP propuesta, lo que corresponde al cuarto de los objetivos específicos del presente trabajo, se plantea que esté ubicada en el organigrama de la compañía entre la gerencia general de la cual dependerá y reportará directamente; y de las tres unidades de negocio con el fin de dar soporte, mejorar el control y el desempeño de los 
diferentes proyectos buscando el cumplimiento y aporte a los compromisos y objetivos estratégicos de la empresa a través del fortalecimiento de la cultura y prácticas de gestión de proyectos, programas y portafolios.

Respondiendo a la cuarta y última pregunta de investigación, relacionada con la manera en la que se puede implementar la centralización de proyectos; se espera que un plazo de máximo dos años, se logre estandarizar y potenciar los procesos de planeación, ejecución y control de sus proyectos e iniciativas; adicional y pensando en aumentar su nivel de madurez en gestión de proyectos, tendrá como objetivo llevar a MYV del nivel 1 - lenguaje común a estar trabajando y fortalecer habilidades en el nivel 3 - metodología propia. Se propone que el tiempo de implementación de la OGP tenga una duración inicial de 12 meses y que se desarrolle en 4 etapas o fases buscando una óptima transición entre el diseño y aprobación, la implementación y puesta en producción de la misma; con esto se da alcance al último de los objetivos específicos planteados el cual corresponde a la elaboración y presentación del plan de implementación de la OGP.

Como recomendaciones, es de gran importancia vincular durante el proceso de recolección de información a varios niveles de la organización, esto permitirá poder contrastar diferentes puntos de vista y posiciones de temas y problemas en común. Adicional a lo anterior, para lograr una aplicación profunda y efectiva de las soluciones propuestas para la organización en donde se desarrolle el trabajo de aplicación empresarial, es necesario conocer y entender las diferentes posiciones y expectativas de la alta gerencia de la empresa; ya que es indispensable que todas las soluciones y planes a desarrollar, estén alineados con las necesidades y objetivos estratégicos. De igual manera, el contar con la aprobación y participación activa de la alta gerencia facilitará la asignación de recursos, resolución de problemas y disponibilización de información requerida. A nivel de sistemas, el utilizar herramientas de análisis estadístico durante la etapa de revisión de resultados, facilitará el correcto entendimiento e interpretación de la data e información recolectada a través de los diferentes instrumentos aplicados durante el desarrollo del trabajo de aplicación.

\section{Agradecimientos}

Se agradece la colaboración de la organización estudiada MYV, así como la gran participación de la Dra. Dora Ariza en el desarrollo de esta investigación.

\section{Referencias}

Ariza, D.A. (2017a). Efectividad de la gestión de los proyectos: una perspectiva constructivista. Obras y Proyectos 22, 75-85

Ariza, D.A. (2017b). Construcción de un modelo de cultura organizacional en el ámbito de los proyectos y su aplicación al sector de tecnología de la información en Colombia. Tesis doctoral, Universidad EAN, Bogotá, Colombia

Casey, W. and Peck, W. (2001). Choosing the right PMO setup. PM Network 15(2), 40-47

Crawford, J.K. and Cabanis-Brewin, J. (2005). Optimizing human capital with a strategic project office: select, train, measure, and reward people for organization success. Auerbach Publications - CRC Press

Hair, J.F., Black, W.C., Babin, B.J. and Anderson, R.E. (2013). Multivariate data analysis. Pearson

Hernández, R.F. (2017). Metodología de la investigación. McGraw-Hill, Colombia

Hernández, R., Fernández, C. y Baptista, P. (2014). Metodología de la investigación. Sexta edición, McGraw-Hill, México

Hill, G.M. (2008). The complete project management office handbook. Auerbach Publications - Taylor \& Francis Group

Hobbs, B. and Aubry, M. (2010). The Project Management Office (PMO): A quest for understanding. PMI Publications, Pennsylvania, USA

Kerzner, H. (2015). Project management 2.0. Wiley \& Sons, New Jersey, USA

Kerzner, H. (2002). Strategic planning for project management using a project management maturity model. John Wiley \& Sons, New York, USA

Kwak, Y.H. and Ibbs, W.C. (2000). The Berkeley project management process maturity model: measuring the value of project management. Proceedings of the 2000 IEEE Engineering Management Society EMS-2000 (Cat. No. 00CH37139), 1-5 
Lewis-Beck, M., Bryman, A.E. and Liao, T.F. (2003). The Sage encyclopedia of social science research methods. Sage Publications, USA

Man, T.J. (2007). A framework for the comparison of maturity models for project-based management. Master thesis, Utrecht University, The Netherlands

PMI (2017). A guide to the project management body of knowledge. Sixth edition. Project Management Institute, Pennsylvania, USA
PMI (2008). Organizational project management maturity model - OPM3®. Project Management Institute, Pennsylvania, USA

Podsakoff, P.M., MacKenzie, S.B., Lee, J.Y. and Podsakoff, N.P. (2003). Common method biases in behavioral research: A critical review of the literature and recommended remedies. Journal of Applied Psychology 88(5), 879-903

Schein, E. (1992). Cultura organizacional y liderazgo. Jossey \& Bass. San Francisco, USA 